\title{
Historia de la Ginecobstetricia en Colombia
}

\author{
PEDRO NEL CARDONA CORREA
}

Por Fernando Sánchez-Torres*

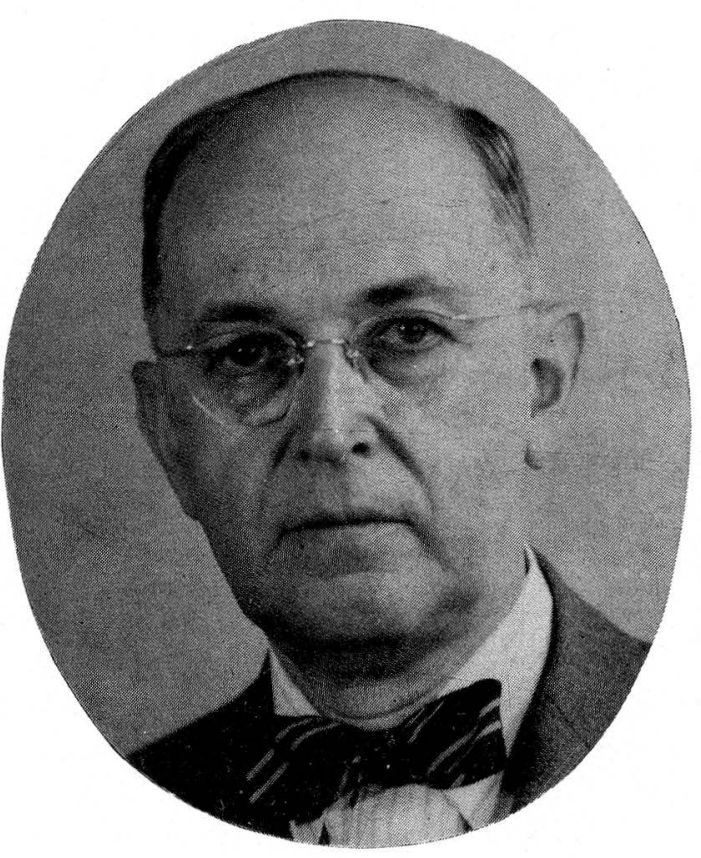

E1 15 de octubre de 1970, al cumplirse el primer aniversario de la muerte del profesor Pedro Nel Cardona Correa, un distinguido grupo de ginecobstetras antioqueños creó en Medellín la Fundación que lleva su nombre, destinada a promover y auspiciar las investigaciones en el campo de la ginecología, disciplina ésta de la que el doctor Cardona fue un gran maestro. Ese grupo de especialistas, casi todos discípulos suyos, quería con ello testimoniarle gratitud a su memoria y, a la vez, perpetuar su nombre. Ese acto, sin duda, fue un elemental reconocimiento a la obra de quien fuera una figura destacada de la ginecología colombiana.

Profesor Titular Honorario de la Universidad Nacional de Colombia. Miembro Fundador de la Sociedad Colombiana de Historia de la Medicina. 
Pedro Nel Cardona nació en Envigado el 24 de septiembre de 1890, del matrimonio compuesto por Tristán Cardona y Ana Joaquina Correa. Sus primeros estudios los adelantó en su ciudad natal; luego ingresó a la Universidad de Antioquia para cursar medicina, recibiéndose el 16 de junio de 1918 con la tesis "Contribución al estudio de la mortalidad infantil”. Seguidamente ocupó las cátedras de Química Biológica y Fisiología. Viajó más tarde a Europa para estudiar anatomía patológica en París, fundando a su regreso dicha cátedra.

En la década de los años 20 la cátedra de Cirugía tenía en la Universidad de Antioquia dos servicios: el de Cirugía General y el de Ginecología, regentados por el profesor Juan B. Montoya y Flórez, considerado “el más grande cirujano de Colombia". Como solía suceder en aquellas calendas, la ginecología apenas comenzaba a adquirir perfil propio y continuaba siendo patrimonio de los cirujanos generales, pues la concepción que de ella se tenía era puramente quirúrgica. De ahí que en Medellín, al igual que en Bogotá, la ginecología, para efectos docentes y asistenciales, formara parte de la cirugía en los programas académicos y en los servicios hospitalarios. Montoya y Flórez había sido el fundador de la cátedra de Clínica Quirúrgica en la Universidad de Antioquia en 1901. Desde entonces sus grandes dotes de cirujano le fueron creando un ambiente de privilegio en los círculos médicos nacionales e internacionales. En la primera década de nuestro siglo practicó 59 histerectomías vaginales, sin un solo fracaso, lo cual es digno de admirar si se tiene en cuenta que para entonces esa era una intervención que apenas comenzaba a ejecutarse entre nosotros. Montoya y Flórez llevó a cabo todo tipo de operaciones con la misma maestría. Era tal su calidad quirúrgica que en 1916, estando en Rochester, fue invitado por los hermanos Mayo para que hiciera algunas demostraciones ante ellos y sus alumnos.

A su regreso de París el doctor Cardona se vinculó nuevamente con la Universidad de Antioquia como docente, esta vez en las cátedras de Anatomía Patológica y de Cancerología. Cuando en 1928 se independizó la Ginecología pasó a ocupar en ella la jefatura de clínica bajo la dirección del también extraordinario cirujano Gil J. Gil, discípulo y continuador de la obra de Montoya y Flórez. Fue profesor titular de Clínica Quirúrgica. Se dice que llegó a practicar 10.000 intervenciones; con razón se le llamaba "el Bisturí de Oro”. Fue él quien de primero ejecutó en Medellín la operación cesárea segmentaria, en 1923.

En el servicio de Ginecología, al lado de Gil J. Gil, Pedro Nel Cardona se encontró a gusto y allí se quedó y echó raíces. Pero su mentalidad no era puramente quirúrgica, no obstante haber trajinado en el campo de la anatomía patológica y de la cancerología. Fue un buen cirujano ginecológico, es cierto, pero fue mejor ginecólogo clínico. Revisando sus publicaciones científicas salta a la vista su interés inicial por la cirugía: tratamiento de la inversión uterina, del prolapso genital, de las malposiciones uterinas, del cáncer del cuello uterino, etc. Más tarde sólo ocupan su atención los temas funcionales: mastopatías, el hiperestrogenismo, la disfunción suprarrenogonadal, la poliquistosis ovárica. Para él la exéresis no era todo. El medio interior de Claude Bernard, la endocrinología, era el verdadero sustrato de la ginecología. Consideraba, con sobrada razón, que todo lo anormal que ocurriera en el aparato genital femenino tenía su correspondiente expresión en ese mundo fabuloso de la célula bajo el influjo de las secreciones internas, es decir, de las hormonas.

Al profesor Cardona le debe la ginecología colombiana el haberla relacionado con la anatomía patológica, no solamente tumoral sino también funcional. Recordemos que él introdujo en Medellín la radioterapia para las neoplasias ginecológicas, con biopsia previa a su aplicación. Comentando esa actuación decía: "Por azar me correspondió la iniciativa de traer la biopsia a Medellín, como elemento previo indispensable para la aplicación de radium en el tratamiento del cáncer ginecológico. Es natural que este he- 
cho, al parecer simple, contribuyó eficazmente a la transformación de la medicina en Antioquia y tal vez por asociación de ideas, se explica mi devoción a la Anatomía Patológica, que en la cátedra de Ginecología ha sido mi faro, mi guión, mi sostén irremplazable en la trayectoria científica del servicio".

En 1938 fue promovido a profesor de Ginecología y designado jefe de la Clínica Ginecológica, adelantando desde esta posición una fecunda labor: implantó el uso de las historias clínicas; estableció la consulta externa, como también la de esterilidad e infertilidad; formó las primeras instrumentadoras quirúrgicas de Antioquia. Después de 45 años de actividad docente, la Universidad de Antioquia, en 1963, le dio el título de Profesor Emérito. Ya antes había sido distinguido por su Alma Mater con la Orden del Mérito Universitario "Francisco Antonio Zea". El doctor Cardona en dos ocasiones fue secretario de la Facultad de Medicina y posteriormente su decano. Presidió también en dos oportunidades la Academia de Medicina de Medellín y fue el primer presidente del Colegio Colombiano de Cirujanos.

Sus últimos días, ya casi octogenario, no fueron entregados al descanso sino que los utilizaba en plan de maestro, es decir, ejerciendo la que fue su profesión espiritual. Tuvo, al igual que el profesor José del Carmen Acosta - su par en el campo de la tocología-, la virtud del discipulismo. En Medellín fundó una verdadera escuela de ginecología, con identidad y respetabilidad, a cuya sombra se formó una pléyade de distinguidos especialistas, entre ellos su propio hijo Fernando. No hay duda de que a Pedro Nel Cardona Correa debe la ginecología. antioqueña su nacimiento científico, como también la afirmación de los cimientos sobre los cuales se destaca en el panorama médico nacional.

\section{BIBLIOGRAFIA}

BONILlA-NAAR, A. Precursores de la Cirugía en Colombia. Antares, Bogotá, p. 45, 1954.

CARDONA, F. Comunicación personal, Medellín, 1976.

CARDONA, P.N. “Inversión uterina”. Clínica Gil, Medellín, 2: 190. 1925.

_— “Histerectomía abdominal para ruptura uterina”. Clínica Gil, Medellín, 3: 421, 1926.

—— “Cáncer del cuello uterino”. Boletín Clínico, Medellín, 4: 243, 1938.

——Desviaciones uterinas y su tratamiento". Boletín Clínico, Medellín, 8: 140, 1942.

—_ “Clínica Ginecológica. (Informe del año 1945)”. Boletín Clínico, Medellín, 18: 11, 1946.

“Tratamiento quirúrgico del prolapso genital". Antioquia Médica, Medellín, 3: 374, 1955.

“Endometriosis" Rev. Colombiana Obst. y Gin. 5: 133, 1954.

- ISAZA, G.; VASQUEZ, G.; CARDONA, F. “Algunas consideraciones al tema de las mastopatías”. Rev. Colombiana Obst. y Gin. 9: 409, 1958.

ISAZA, G.; CARDONA, F. "Pronóstico precoz del tratamiento del cáncer uterino por medio de la citología”. Rev. Colombiana Obst. y Gin. 10: 327, 1959.

ISAZA, G.; CARDONA, F. “Las micosis en las leucorreas”. Rev. Colombiana Obst. y Gine. 11: 130, 1960. 
GUZMAN, A.; ISAZA, S. y cols. "Comentario sobre flujos genitales". Rev. Colombiana Obst. y Ginc. 11: $101,1960$.

CARDONA, F.; ZUNIGA, R. "Dosificación de los estrógenos en la orina". Rev. Colombiana Obst. y Gin. 11: $50,1960$.

DIAZ, R.; ISAZA, G.; VASQUEZ, G.; CARDONA, F. "Hiperestrogenismo”. Rev. Colombiana Obst. y Gin. 11: $1,1960$.

CARDONA, F.; ZUÑIGA, R. "Valoración del F.S.H. por la citología”. Rev. Colombiana Obst. y Gin. 11: 401,1960 .

CARDONA, F.; ZUNIGA, R. "Los 17 cetoesteroides fraccionados en ginecología.". Rev. Colombiana de Obstetricia y Ginecología. 13: 333, 1962.

CARDONA, F.; ZUÑIGA, R. "Disfunción suprarrenogonadal en el homosexualismo y su tratamiento". Rev. Colombiana Obst. y Gin. 13: 320, 1962.

GUZMAN, A.; CARDONA, F. y cols. “Amibiasis genital femenina”. Rev. Colombiana Obst. y Gin. 13: $37,1962$.

“Discurso con ocasión de su retiro de la Cátedra de Ginecología” Antioquia Médica 13: 164, 1963.

BEDOYA, V.; CARDONA, F. y cols. “Poliquistosis ovárica”. Rev. Colombiana Obst. y Gin. 19: 171, 1968. 